\title{
MINERALS IN BEETROOT
}

\section{3}

\begin{tabular}{|c|c|c|c|c|c|c|c|c|c|c|c|c|c|c|c|c|c|c|c|}
\hline \multirow[t]{2}{*}{ ID } & \multirow{2}{*}{$\begin{array}{c}\text { sample } \\
\text { label } \\
\end{array}$} & \multirow[b]{2}{*}{ var } & \multirow[b]{2}{*}{ rep } & \multirow[b]{2}{*}{ rep } & \multirow{2}{*}{$\begin{array}{l}\text { yield } \\
\text { t/ha }\end{array}$} & \multirow{2}{*}{$\begin{array}{c}\% \\
\text { ST } \\
\end{array}$} & \multirow{2}{*}{\begin{tabular}{|c|} 
crude \\
proteins \\
\end{tabular}} & \multicolumn{7}{|c|}{$\%$ in dry matter } & \multicolumn{4}{|c|}{$\mathrm{mg} / \mathrm{kg}$ in dry matter } & \multirow{2}{*}{$\begin{array}{c}\% \\
\text { Brix } \\
\end{array}$} \\
\hline & & & & & & & & $\mathbf{N}$ & $\mathrm{P}_{2} \mathrm{O}_{5}$ & $\mathbf{P}$ & $\mathrm{K}_{2} \mathrm{O}$ & $\mathrm{K}$ & $\mathrm{Ca}$ & $\mathrm{Mg}$ & $\mathrm{Fe}$ & $\mathrm{Zn}$ & $\mathrm{Mn}$ & $\mathrm{Cu}$ & \\
\hline & oznaka za SAS & var & rep1 & rep2 & prinos_03 & ST_03 & sirprot_03 & N_03 & $\begin{array}{l}\text { P2O5_03 } \\
\end{array}$ & P_03 & K2O_03 & K_03 & Ca_03 & Mg_03 & $\mathrm{Fe}_{2} 03$ & $2 \mathrm{Zn} \_03$ & Mn_03 & Cu_03 & Brix_03 \\
\hline 1398 & 1 & 1 & $\mathrm{I}$ & $\mathrm{I}$ & 1,2 & 7,5 & 16,8 & 2,68 & 0,38 & 0,17 & 2,98 & 2,47 & 0,46 & 0,61 & 300 & 48 & 99 & 11,6 & 7,0 \\
\hline 1399 & 2 & 2 & 1 & II & 10,6 & 6,2 & 17,7 & 2,83 & 0,48 & 0,21 & 2,78 & 2,31 & 0,4 & 0,63 & 290 & 43 & 75 & 10,6 & 9,0 \\
\hline 1400 & 3 & 3 & $\mathbf{I}$ & III & 6,0 & 6,5 & 17,1 & 2,74 & 0,43 & 0,19 & 2,96 & 2,46 & 0,42 & 0,51 & 180 & 41 & 97 & 11,0 & 8,0 \\
\hline 1401 & 4 & 4 & I & IV & 10,1 & 6,4 & 19,1 & 3,06 & 0,46 & 0,20 & 3,62 & 3,00 & 0,41 & 0,56 & 170 & 44 & 110 & 11,8 & 10,0 \\
\hline 1402 & 5 & 2 & II & IV & 8,0 & 5,5 & 24,2 & 3,87 & 0,56 & 0,24 & 3,92 & 3,25 & 0,52 & 0,8 & 200 & 62 & 89 & 12,8 & 8,0 \\
\hline 1403 & 6 & 4 & II & III & 10,3 & 7,1 & 17,5 & 2,8 & 0,28 & 0,12 & 3,00 & 2,49 & 0,3 & 0,46 & 230 & 40 & 87 & 11,0 & 7,0 \\
\hline 1404 & 7 & 1 & II & II & 4,4 & 5,8 & 21,5 & 3,44 & 0,49 & 0,21 & 3,62 & 3,00 & 0,36 & 0,8 & 269 & 66 & 100 & 12,0 & 7,0 \\
\hline 1405 & 8 & 3 & II & 1 & 6,1 & 6,8 & 19,6 & 3,14 & 0,5 & 0,22 & 3,62 & 3,00 & 0,42 & 0,66 & 300 & 55 & 106 & 11,0 & 5,0 \\
\hline 1406 & 9 & 2 & III & 1 & 12,9 & 6,6 & 20,1 & 3,22 & 0,62 & 0,27 & 3,72 & 3,09 & 0,53 & 0,7 & 310 & 38 & 80 & 12,0 & 7,0 \\
\hline 1407 & 10 & 4 & III & II & 6,7 & 7,3 & 21,8 & 3,48 & 0,51 & 0,22 & 3,99 & 3,31 & 0,38 & 0,71 & 300 & 54 & 98 & 12,1 & 6,0 \\
\hline 1408 & 11 & 1 & III & III & 3,0 & 7,9 & 20,3 & 3,24 & 0,48 & 0,21 & 3,98 & 3,30 & 0,36 & 0,69 & 370 & 56 & 112 & 11,0 & 8,0 \\
\hline 1409 & 12 & 3 & III & IV & 2,8 & 6,5 & 23,4 & 3,75 & 0,46 & 0,20 & 3,72 & 3,09 & 0,4 & 0,69 & 440 & 67 & 118 & 13,2 & 7,0 \\
\hline 1410 & 13 & 1 & IV & IV & 3,9 & 7,3 & 22,6 & 3,61 & 0,46 & 0,20 & 3,10 & 2,57 & 0,45 & 0,66 & 300 & 69 & 114 & 13,2 & 5,0 \\
\hline 1411 & 14 & 2 & IV & III & 8,3 & 6,3 & 23,6 & 3,77 & 0,81 & 0,35 & 3,60 & 2,99 & 0,49 & 0,76 & 269 & 57 & 86 & 10,9 & 5,0 \\
\hline 1412 & 15 & 3 & IV & II & 8,6 & 7,2 & 19,4 & 3,1 & 0,44 & 0,19 & 2,86 & 2,37 & 0,36 & 0,61 & 190 & 58 & 80 & 11,2 & 8,0 \\
\hline \multirow[t]{2}{*}{1413} & 16 & 4 & IV & 1 & 5,6 & 7,0 & 23,1 & 3,7 & 0,45 & 0,20 & 2,64 & 2,19 & 0,53 & 0,64 & 190 & 50 & 80 & 12,6 & 6,0 \\
\hline & & & & & yield & $\%$ & crude & \multicolumn{7}{|c|}{$\%$ in dry matter } & \multicolumn{4}{|c|}{$\mathrm{mg} / \mathrm{kg}$ in dry matter } & $\%$ \\
\hline \multicolumn{3}{|c|}{ Average by variants } & & & t/ha & ST & proteins & $\mathbf{N}$ & $\mathrm{P}_{2} \mathrm{O}_{5}$ & $\mathbf{P}$ & $\mathrm{K}_{2} \mathrm{O}$ & K & $\mathrm{Ca}$ & $\mathrm{Mg}$ & $\mathrm{Fe}$ & $\mathrm{Zn}$ & $\mathrm{Mn}$ & $\mathrm{Cu}$ & Brix \\
\hline varijant 1 & unfertilized & ontrol & & & 3,1 & 7,1 & 20,3 & 3,2 & 0,5 & 0,20 & 3,4 & 2,84 & 0,4 & 0,7 & 310 & 60 & 106 & 12,0 & 6,8 \\
\hline varijant 2 & \multicolumn{3}{|c|}{ stable manure, $50 \mathrm{t} / \mathrm{ha}$} & & 9,9 & 6,2 & 21,4 & 3,4 & 0,6 & 0,27 & 3,5 & 2,91 & 0,5 & 0,7 & 267 & 50 & 83 & 11,6 & 7,3 \\
\hline varijant 3 & \multicolumn{2}{|c|}{500 kg NPK/ha* } & & & 5,9 & 6,7 & 19,9 & 3,2 & 0,5 & 0,20 & 3,3 & 2,73 & 0,4 & 0,6 & 278 & 55 & 100 & 11,6 & 7,0 \\
\hline varijant 4 & \multicolumn{2}{|c|}{1000 kg NPK/ha* } & & & 8,2 & 6,9 & 20,4 & 3,3 & 0,4 & 0,19 & 3,3 & 2,75 & 0,4 & 0,6 & 223 & 47 & 94 & 11,9 & 7,3 \\
\hline
\end{tabular}




\section{MINERALS IN BEETROOT}

\section{3}

\begin{tabular}{|c|c|c|c|c|c|c|c|c|c|c|c|c|c|c|c|c|c|c|c|}
\hline \multirow[t]{2}{*}{ ID } & \multirow{2}{*}{$\begin{array}{c}\text { sample } \\
\text { label } \\
\end{array}$} & \multirow[b]{2}{*}{ var } & \multirow[b]{2}{*}{ rep } & \multirow[b]{2}{*}{ rep } & \multirow{2}{*}{$\begin{array}{l}\text { yield } \\
\text { t/ha } \\
\end{array}$} & \multirow{2}{*}{$\begin{array}{l}\% \\
\text { ST }\end{array}$} & \multirow{2}{*}{\begin{tabular}{|c|} 
crude \\
proteins \\
\end{tabular}} & \multicolumn{7}{|c|}{$\%$ in fresh matter } & \multicolumn{4}{|c|}{$\mathrm{mg} / \mathrm{kg}$ in fresh matter } & \multirow{2}{*}{$\begin{array}{c}\% \\
\text { Brix } \\
\end{array}$} \\
\hline & & & & & & & & $\mathbf{N}$ & $\mathrm{P}_{2} \mathrm{O}_{5}$ & $\mathbf{P}$ & $\mathrm{K}_{2} \mathrm{O}$ & $\mathbf{K}$ & $\mathrm{Ca}$ & $\mathrm{Mg}$ & $\mathrm{Fe}$ & $\mathrm{Zn}$ & $\mathrm{Mn}$ & $\mathrm{Cu}$ & \\
\hline & oznaka za SAS & var & rep1 & rep2 & prinos_03 & ST_03 & sirprot_03 & N_03 & P205_03 & P_03 & K2O_03 & K_03 & $\mathrm{Ca} 03$ & Mg_03 & $\mathrm{Fe}_{-} 03$ & $2 n \_03$ & Mn_03 & Cu_03 & Brix_03 \\
\hline 1398 & 1 & 1 & 1 & 1 & 1,2 & 7,5 & 1,2 & 0,20 & 0,03 & 0,01 & 0,22 & 0,18 & 0,03 & 0,05 & 22,35 & 3,58 & 7,38 & 0,86 & 7,0 \\
\hline 1399 & 2 & 2 & $I$ & II & 10,6 & 6,2 & 1,1 & 0,18 & 0,03 & 0,01 & 0,17 & 0,14 & 0,02 & 0,04 & 18,07 & 2,68 & 4,67 & 0,66 & 9,0 \\
\hline 1400 & 3 & 3 & $\mathbf{I}$ & III & 6,0 & 6,5 & 1,1 & 0,18 & 0,03 & 0,01 & 0,19 & 0,16 & 0,03 & 0,03 & 11,75 & 2,68 & 6,33 & 0,72 & 8,0 \\
\hline 1401 & 4 & 4 & $I$ & IV & 10,1 & 6,4 & 1,2 & 0,19 & 0,03 & 0,01 & 0,23 & 0,19 & 0,03 & 0,04 & 10,80 & 2,79 & 6,99 & 0,75 & 10,0 \\
\hline 1402 & 5 & 2 & II & IV & 8,0 & 5,5 & 1,3 & 0,21 & 0,03 & 0,01 & 0,22 & 0,18 & 0,03 & 0,04 & 11,08 & 3,43 & 4,93 & 0,71 & 8,0 \\
\hline 1403 & 6 & 4 & II & III & 10,3 & 7,1 & 1,2 & 0,20 & 0,02 & 0,01 & 0,21 & 0,18 & 0,02 & 0,03 & 16,31 & 2,84 & 6,17 & 0,78 & 7,0 \\
\hline 1404 & 7 & 1 & II & II & 4,4 & 5,8 & 1,2 & 0,20 & 0,03 & 0,01 & 0,21 & 0,17 & 0,02 & 0,05 & 15,60 & 3,83 & 5,80 & 0,70 & 7,0 \\
\hline 1405 & 8 & 3 & II & 1 & 6,1 & 6,8 & 1,3 & 0,21 & 0,03 & 0,01 & 0,24 & 0,20 & 0,03 & 0,04 & 20,28 & 3,72 & 7,17 & 0,74 & 5,0 \\
\hline 1406 & 9 & 2 & III & 1 & 12,9 & 6,6 & 1,3 & 0,21 & 0,04 & 0,02 & 0,25 & 0,20 & 0,03 & 0,05 & 20,43 & 2,50 & 5,27 & 0,79 & 7,0 \\
\hline 1407 & 10 & 4 & III & II & 6,7 & 7,3 & 1,6 & 0,25 & 0,04 & 0,02 & 0,29 & 0,24 & 0,03 & 0,05 & 21,96 & 3,95 & 7,17 & 0,89 & 6,0 \\
\hline 1408 & 11 & 1 & III & III & 3,0 & 7,9 & 1,6 & 0,26 & 0,04 & 0,02 & 0,32 & 0,26 & 0,03 & 0,05 & 29,38 & 4,45 & 8,89 & 0,87 & 8,0 \\
\hline 1409 & 12 & 3 & III & IV & 2,8 & 6,5 & 1,5 & 0,24 & 0,03 & 0,01 & 0,24 & 0,20 & 0,03 & 0,04 & 28,38 & 4,32 & 7,61 & 0,85 & 7,0 \\
\hline 1410 & 13 & 1 & IV & IV & 3,9 & 7,3 & 1,6 & 0,26 & 0,03 & 0,01 & 0,23 & 0,19 & 0,03 & 0,05 & 21,87 & 5,03 & 8,31 & 0,96 & 5,0 \\
\hline 1411 & 14 & 2 & IV & III & 8,3 & 6,3 & 1,5 & 0,24 & 0,05 & 0,02 & 0,23 & 0,19 & 0,03 & 0,05 & 16,97 & 3,60 & 5,43 & 0,69 & 5,0 \\
\hline 1412 & 15 & 3 & IV & II & 8,6 & 7,2 & 1,4 & 0,22 & 0,03 & 0,01 & 0,20 & 0,17 & 0,03 & 0,04 & 13,59 & 4,15 & 5,72 & 0,80 & 8,0 \\
\hline \multirow[t]{2}{*}{1413} & 16 & 4 & IV & 1 & 5,6 & 7,0 & 1,6 & 0,26 & 0,03 & 0,01 & 0,18 & 0,15 & 0,04 & 0,04 & 13,26 & 3,49 & 5,58 & 0,88 & 6,0 \\
\hline & & & & & yield & $\%$ & crude & \multicolumn{7}{|c|}{$\%$ in fresh matter } & \multicolumn{4}{|c|}{$\mathrm{mg} / \mathrm{kg}$ in fresh matter } & $\%$ \\
\hline \multicolumn{3}{|c|}{ Average by variants } & & & t/ha & ST & proteins & $\mathbf{N}$ & $\mathrm{P}_{2} \mathrm{O}_{5}$ & $\mathbf{P}$ & $\mathrm{K}_{2} \mathrm{O}$ & K & $\mathrm{Ca}$ & $\mathrm{Mg}$ & $\mathrm{Fe}$ & $\mathrm{Zn}$ & $\mathrm{Mn}$ & $\mathrm{Cu}$ & Brix \\
\hline varijant 1 & \multirow{2}{*}{\multicolumn{3}{|c|}{\begin{tabular}{|l|l} 
unfertilized control \\
stable manure, $50 \mathrm{t} / \mathrm{ha}$ \\
\end{tabular}}} & & 3,1 & 7,1 & 1,4 & 0,2 & 0,03 & 0,01 & 0,2 & 0,2 & 0,0 & 0,0 & 22 & 4 & 8 & 0,8 & 6,8 \\
\hline varijant 2 & & & & & 9,9 & 6,2 & 1,3 & 0,2 & 0,04 & 0,02 & 0,2 & 0,2 & 0,0 & 0,0 & 17 & 3 & 5 & 0,7 & 7,3 \\
\hline varijant 3 & \multicolumn{2}{|c|}{500 kg NPK/ha* } & & & 5,9 & 6,7 & 1,3 & 0,2 & 0,03 & 0,01 & 0,2 & 0,2 & 0,0 & 0,0 & 18 & 4 & 7 & 0,8 & 7,0 \\
\hline varijant 4 & \multicolumn{2}{|c|}{1000 kg NPK/ha* } & & & 8,2 & 6,9 & 1,4 & 0,2 & 0,03 & 0,01 & 0,2 & 0,2 & 0,0 & 0,0 & 16 & 3 & 6 & 0,8 & 7,3 \\
\hline
\end{tabular}


formula: broj*1000

MINERALS IN BEETROOT makroelementi: formula: broj*1000 mikroelementi: formula: broj/10

\section{3}

\begin{tabular}{|c|c|c|c|c|c|c|c|c|c|c|c|c|c|c|c|c|c|c|c|}
\hline \multirow[t]{2}{*}{ ID } & \multirow{2}{*}{$\begin{array}{c}\text { sample } \\
\text { label } \\
\end{array}$} & \multirow[b]{2}{*}{ var } & \multirow[b]{2}{*}{ rep } & \multirow[b]{2}{*}{ rep } & \multirow{2}{*}{$\begin{array}{l}\text { yield } \\
\text { t/ha } \\
\end{array}$} & \multirow{2}{*}{$\begin{array}{l}\% \\
S T \\
\end{array}$} & \multirow{2}{*}{\begin{tabular}{|c|} 
crude \\
proteins \\
\end{tabular}} & \multicolumn{7}{|c|}{$\mathrm{mg} / 100 \mathrm{~g}$ in fresh matter } & \multicolumn{4}{|c|}{$\mathrm{mg} / 100 \mathrm{~g}$ in fresh matter } & \multirow{2}{*}{$\begin{array}{c}\% \\
\text { Brix } \\
\end{array}$} \\
\hline & & & & & & & & $\mathbf{N}$ & $\mathrm{P}_{2} \mathrm{O}_{5}$ & $\mathbf{P}$ & $\mathrm{K}_{2} \mathrm{O}$ & K & $\mathrm{Ca}$ & $\mathrm{Mg}$ & $\mathrm{Fe}$ & $\mathrm{Zn}$ & $\mathrm{Mn}$ & $\mathrm{Cu}$ & \\
\hline & oznaka za SAS & var & rep1 & rep2 & prinos_03 & ST_03 & \begin{tabular}{|l|} 
sirprot_03 \\
\end{tabular} & N_03 & P205_03 & P_03 & K2O_03 & K_03 & $\mathrm{Ca} 03$ & Mg_03 & $\mathrm{Fe}_{-} 03$ & $2 n_{-} 03$ & Mn_03 & Cu_03 & Brix_03 \\
\hline 1398 & 1 & 1 & $\mathbf{I}$ & $\mathrm{I}$ & 1,2 & 7,5 & 1247,9 & 199,7 & 28,3 & 12,4 & 222,0 & 184,2 & 34,3 & 45,4 & 2,24 & 0,36 & 0,74 & 0,09 & 7,0 \\
\hline 1399 & 2 & 2 & $\mathbf{I}$ & II & 10,6 & 6,2 & 1101,9 & 176,3 & 29,9 & 13,1 & 173,2 & 143,7 & 24,9 & 39,2 & 1,81 & 0,27 & 0,47 & 0,07 & 9,0 \\
\hline 1400 & 3 & 3 & $I$ & III & 6,0 & 6,5 & 1118,3 & 178,9 & 28,1 & 12,3 & 193,3 & 160,4 & 27,4 & 33,3 & 1,18 & 0,27 & 0,63 & 0,07 & 8,0 \\
\hline 1401 & 4 & 4 & $I$ & IV & 10,1 & 6,4 & 1214,4 & 194,3 & 29,2 & 12,8 & 229,9 & 190,8 & 26,0 & 35,6 & 1,08 & 0,28 & 0,70 & 0,07 & 10,0 \\
\hline 1402 & 5 & 2 & II & IV & 8,0 & 5,5 & 1340,0 & 214,4 & 31,0 & 13,5 & 217,2 & 180,2 & 28,8 & 44,3 & 1,11 & 0,34 & 0,49 & 0,07 & 8,0 \\
\hline 1403 & 6 & 4 & II & III & 10,3 & 7,1 & 1240,8 & 198,5 & 19,9 & 8,7 & 212,7 & 176,5 & 21,3 & 32,6 & 1,63 & 0,28 & 0,62 & 0,08 & 7,0 \\
\hline 1404 & 7 & 1 & II & II & 4,4 & 5,8 & 1247,0 & 199,5 & 28,4 & 12,4 & 210,0 & 174,2 & 20,9 & 46,4 & 1,56 & 0,38 & 0,58 & 0,07 & 7,0 \\
\hline 1405 & 8 & 3 & II & 1 & 6,1 & 6,8 & 1326,7 & 212,3 & 33,8 & 14,8 & 244,7 & 203,1 & 28,4 & 44,6 & 2,03 & 0,37 & 0,72 & 0,07 & 5,0 \\
\hline 1406 & 9 & 2 & III & 1 & 12,9 & 6,6 & 1326,2 & 212,2 & 40,9 & 17,8 & 245,1 & 203,4 & 34,9 & 46,1 & 2,04 & 0,25 & 0,53 & 0,08 & 7,0 \\
\hline 1407 & 10 & 4 & III & II & 6,7 & 7,3 & 1592,1 & 254,7 & 37,3 & 16,3 & 292,1 & 242,4 & 27,8 & 52,0 & 2,20 & 0,40 & 0,72 & 0,09 & 6,0 \\
\hline 1408 & 11 & 1 & III & III & 3,0 & 7,9 & 1607,9 & 257,3 & 38,1 & 16,6 & 316,0 & 262,3 & 28,6 & 54,8 & 2,94 & 0,44 & 0,89 & 0,09 & 8,0 \\
\hline 1409 & 12 & 3 & III & IV & 2,8 & 6,5 & 1511,7 & 241,9 & 29,7 & 13,0 & 239,9 & 199,1 & 25,8 & 44,5 & 2,84 & 0,43 & 0,76 & 0,09 & 7,0 \\
\hline 1410 & 13 & 1 & IV & IV & 3,9 & 7,3 & 1644,8 & 263,2 & 33,5 & 14,6 & 226,0 & 187,5 & 32,8 & 48,1 & 2,19 & 0,50 & 0,83 & 0,10 & 5,0 \\
\hline 1411 & 14 & 2 & IV & III & 8,3 & 6,3 & 1486,8 & 237,9 & 51,1 & 22,3 & 227,2 & 188,5 & 30,9 & 48,0 & 1,70 & 0,36 & 0,54 & 0,07 & 5,0 \\
\hline 1412 & 15 & 3 & IV & II & 8,6 & 7,2 & 1385,3 & 221,7 & 31,5 & 13,7 & 204,5 & 169,7 & 25,7 & 43,6 & 1,36 & 0,41 & 0,57 & 0,08 & 8,0 \\
\hline 1413 & 16 & 4 & IV & 1 & 5,6 & 7,0 & 1614,1 & 258,3 & 31,4 & 13,7 & 184,3 & 152,9 & 37,0 & 44,7 & 1,33 & 0,35 & 0,56 & 0,09 & 6,0 \\
\hline
\end{tabular}

\begin{tabular}{|c|c|c|c|c|c|c|c|c|c|c|c|c|c|c|c|c|}
\hline \multirow{3}{*}{\multicolumn{2}{|c|}{ Average by variants }} & \multirow{3}{*}{$\begin{array}{c}\text { yield } \\
\mathrm{t} / \mathrm{ha} \\
\end{array}$} & \multirow{3}{*}{$\begin{array}{l}\% \\
\text { ST } \\
\end{array}$} & \multirow{3}{*}{\begin{tabular}{|c|} 
crude \\
proteins \\
\end{tabular}} & \multirow{2}{*}{\multicolumn{7}{|c|}{$\mathrm{mg} / 100 \mathrm{~g}$ in fresh matter }} & \multirow{2}{*}{\multicolumn{4}{|c|}{$\mathrm{mg} / 100 \mathrm{~g}$ in fresh matter }} & \multirow{3}{*}{$\begin{array}{c}\% \\
\text { Brix } \\
\end{array}$} \\
\hline & & & & & & & & & & & & & & & & \\
\hline & & & & & $\mathbf{N}$ & $\mathbf{P}_{2} \mathbf{O}_{5}$ & $\mathbf{P}$ & $\mathrm{K}_{2} \mathrm{O}$ & $\mathbf{K}$ & $\mathrm{Ca}$ & $\mathrm{Mg}$ & Fe & $\mathrm{Zn}$ & $\mathrm{Mn}$ & $\mathrm{Cu}$ & \\
\hline varijant 1 & unfertilized control & 3,1 & 7,1 & 1437 & 230 & 32 & 14 & 243 & 202 & 29 & 49 & 2,23 & 0,42 & 0,76 & 0,08 & 6,8 \\
\hline varijant 2 & stable manure, $50 \mathrm{t} / \mathrm{ha}$ & 9,9 & 6,2 & 1314 & 210 & 38 & 17 & 216 & 179 & 30 & 44 & 1,66 & 0,31 & 0,51 & 0,07 & 7,3 \\
\hline varijant 3 & 500 kg NPK/ha* & 5,9 & 6,7 & 1335 & 214 & 31 & 13 & 221 & 183 & 27 & 42 & 1,85 & 0,37 & 0,67 & 0,08 & 7,0 \\
\hline varijant 4 & 1000 kg NPK/ha* & 8,2 & 6,9 & 1415 & 226 & 29 & 13 & 230 & 191 & 28 & 41 & 1,56 & 0,33 & 0,65 & 0,08 & 7,3 \\
\hline
\end{tabular}




\section{MINERALS IN BEETROOT}

2004

\begin{tabular}{|c|c|c|c|c|c|c|c|c|c|c|c|c|c|c|c|c|c|c|c|}
\hline \multirow[t]{2}{*}{ ID } & \multirow{2}{*}{$\begin{array}{c}\text { sample } \\
\text { label } \\
\end{array}$} & \multirow[b]{2}{*}{ var } & \multirow[b]{2}{*}{ rep } & \multirow[b]{2}{*}{ rep } & \multirow{2}{*}{$\begin{array}{l}\text { yield } \\
\text { t/ha } \\
\end{array}$} & \multirow{2}{*}{$\begin{array}{l}\% \\
\text { ST } \\
\end{array}$} & \multirow{2}{*}{\begin{tabular}{|c|} 
crude \\
proteins \\
\end{tabular}} & \multicolumn{7}{|c|}{$\%$ in dry matter } & \multicolumn{4}{|c|}{$\mathrm{mg} / \mathrm{kg}$ in dry matter } & \multirow{2}{*}{$\begin{array}{c}\% \\
\text { Brix } \\
\end{array}$} \\
\hline & & & & & & & & $\mathbf{N}$ & $\mathrm{P}_{2} \mathrm{O}_{5}$ & $\mathbf{P}$ & $\mathrm{K}_{2} \mathrm{O}$ & $K$ & $\mathrm{Ca}$ & $\mathrm{Mg}$ & $\mathrm{Fe}$ & $\mathrm{Zn}$ & $\mathrm{Mn}$ & $\mathrm{Cu}$ & \\
\hline & oznaka za SAS & var & rep1 & rep2 & prinos_04 & ST_04 & sirprot_04 & N_04 & $\begin{array}{l}\text { P2O5_04 } \\
\end{array}$ & P_04 & K2O_04 & K_04 & Ca_04 & Mg_04 & $\mathrm{Fe} \_04$ & $2 \mathrm{Zn}$-04 & Mn_04 & Cu_04 & Brix_04 \\
\hline 2091 & 1 & 1 & $\mathbf{I}$ & $\mathrm{I}$ & 37,5 & 15,6 & 9,1 & 1,46 & 0,46 & 0,20 & 2,69 & 2,24 & 0,25 & 0,25 & 120 & 34 & 51 & 8,0 & 13,6 \\
\hline 2094 & 2 & 2 & $I$ & II & 42,7 & 13,0 & 9,7 & 1,55 & 0,69 & 0,30 & 3,38 & 2,82 & 0,21 & 0,20 & 110 & 33 & 40 & 9,5 & 10,5 \\
\hline 2097 & 3 & 3 & $\mathbf{I}$ & III & 44,1 & 15,9 & 10,1 & 1,61 & 0,63 & 0,28 & 3,46 & 2,88 & 0,20 & 0,25 & 99 & 32 & 44 & 9,8 & 12,6 \\
\hline 2100 & 4 & 4 & $I$ & IV & 42,9 & 16,0 & 9,5 & 1,52 & 0,59 & 0,26 & 3,24 & 2,70 & 0,24 & 0,18 & 108 & 28 & 41 & 11,0 & 12,9 \\
\hline 2103 & 5 & 2 & II & IV & 46,6 & 13,2 & 9,3 & 1,49 & 0,77 & 0,34 & 3,57 & 2,98 & 0,23 & 0,24 & 121 & 30 & 36 & 11,0 & 10 \\
\hline 2106 & 6 & 4 & II & III & 52,7 & 14,4 & 11,0 & 1,76 & 0,63 & 0,28 & 3,02 & 2,52 & 0,22 & 0,24 & 99 & 29 & 36 & 9,6 & 11,8 \\
\hline 2109 & 7 & 1 & II & II & 43,1 & 14,5 & 9,7 & 1,55 & 0,59 & 0,26 & 2,76 & 2,30 & 0,20 & 0,21 & 90 & 32 & 40 & 9,6 & 12 \\
\hline 2112 & 8 & 3 & II & 1 & 42,4 & 13,4 & 11,4 & 1,83 & 0,58 & 0,25 & 2,96 & 2,47 & 0,19 & 0,22 & 108 & 37 & 41 & 9,8 & 11,6 \\
\hline 2115 & 9 & 2 & III & 1 & 41,1 & 13,5 & 8,9 & 1,43 & 0,306 & 0,13 & 2,78 & 2,32 & 0,16 & 0,20 & 99 & 28 & 40 & 8,3 & 10,5 \\
\hline 2118 & 10 & 4 & III & II & 49,8 & 13,6 & 9,9 & 1,58 & 0,339 & 0,15 & 2,91 & 2,43 & 0,19 & 0,19 & 99 & 28 & 40 & 8,2 & 11,9 \\
\hline 2121 & 11 & 1 & III & III & 47,3 & 13,9 & 8,8 & 1,41 & 0,296 & 0,13 & 2,74 & 2,28 & 0,34 & 0,28 & 116 & 28 & 43 & 8,8 & 12,3 \\
\hline 2124 & 12 & 3 & III & IV & 46,8 & 15,0 & 7,4 & 1,18 & 0,314 & 0,14 & 2,48 & 2,07 & 0,16 & 0,20 & 92 & 28 & 43 & 6,9 & 13,2 \\
\hline 2127 & 13 & 1 & IV & IV & 36,1 & 15,0 & 8,3 & 1,32 & 0,255 & 0,11 & 2,56 & 2,13 & 0,21 & 0,20 & 108 & 26 & 51 & 9,6 & 13,4 \\
\hline 2130 & 14 & 2 & IV & III & 40,0 & 15,9 & 8,6 & 1,37 & 0,283 & 0,12 & 2,70 & 2,25 & 0,19 & 0,23 & 80 & 26 & 52 & 9,1 & 13,8 \\
\hline 2133 & 15 & 3 & IV & II & 43,2 & 15,6 & 8,4 & 1,34 & 0,278 & 0,12 & 2,57 & 2,14 & 0,18 & 0,18 & 93 & 28 & 49 & 8,9 & 13,2 \\
\hline \multirow[t]{2}{*}{2136} & 16 & 4 & IV & 1 & 38,1 & 18,3 & 8,4 & 1,34 & 0,244 & 0,11 & 2,29 & 1,91 & 0,21 & 0,19 & 90 & 28 & 49 & 8,8 & 14,7 \\
\hline & & & & & yield & $\%$ & crude & \multicolumn{7}{|c|}{$\%$ in dry matter } & \multicolumn{4}{|c|}{$\mathrm{mg} / \mathrm{kg}$ in dry matter } & $\%$ \\
\hline \multicolumn{3}{|c|}{ Average by variants } & & & t/ha & ST & proteins & $\mathbf{N}$ & $\mathrm{P}_{2} \mathrm{O}_{5}$ & $\mathbf{P}$ & $\mathrm{K}_{2} \mathrm{O}$ & $K$ & $\mathrm{Ca}$ & $\mathrm{Mg}$ & $\mathrm{Fe}$ & $\mathrm{Zn}$ & $\mathrm{Mn}$ & $\mathrm{Cu}$ & Brix \\
\hline varijant 1 & \multirow{2}{*}{\multicolumn{3}{|c|}{ stable manure, $50 \mathrm{t} / \mathrm{ha}$}} & & 41,0 & 14,7 & 9,0 & 1,4 & 0,4 & 0,17 & 2,7 & 2,2 & 0,25 & 0,23 & 109 & 30 & 46 & 9,0 & 12,8 \\
\hline varijant 2 & & & & & 42,6 & 13,9 & 9,1 & 1,5 & 0,5 & 0,22 & 3,1 & 2,6 & 0,20 & 0,22 & 103 & 29 & 42 & 9,5 & 11,2 \\
\hline varijant 3 & \multicolumn{2}{|c|}{500 kg NPK/ha* } & & & 44,1 & 15,0 & 9,3 & 1,5 & 0,5 & 0,20 & 2,9 & 2,4 & 0,18 & 0,21 & 98 & 31 & 44 & 8,9 & 12,7 \\
\hline varijant 4 & \multicolumn{2}{|c|}{1000 kg NPK/ha* } & & & 45,9 & 15,6 & 9,7 & 1,6 & 0,5 & 0,20 & 2,9 & 2,4 & 0,21 & 0,20 & 99 & 28 & 42 & 9,4 & 12,8 \\
\hline
\end{tabular}




\section{MINERALS IN BEETROOT}

\section{4}

\begin{tabular}{|c|c|c|c|c|c|c|c|c|c|c|c|c|c|c|c|c|c|c|c|}
\hline \multirow[t]{2}{*}{ ID } & \multirow{2}{*}{$\begin{array}{c}\text { sample } \\
\text { label }\end{array}$} & \multirow[b]{2}{*}{ var } & \multirow[b]{2}{*}{ rep } & \multirow[b]{2}{*}{ rep } & \multirow{2}{*}{$\begin{array}{l}\text { yield } \\
\text { t/ha }\end{array}$} & \multirow{2}{*}{$\begin{array}{l}\% \\
\text { ST } \\
\end{array}$} & \multirow{2}{*}{\begin{tabular}{|c|} 
crude \\
proteins \\
\end{tabular}} & \multicolumn{7}{|c|}{$\%$ in fresh matter } & \multicolumn{4}{|c|}{$\mathrm{mg} / \mathrm{kg}$ in fresh matter } & \multirow{2}{*}{$\begin{array}{c}\% \\
\text { Brix } \\
\end{array}$} \\
\hline & & & & & & & & $\mathbf{N}$ & $\mathbf{P}_{2} \mathbf{O}_{5}$ & $\mathbf{P}$ & $\mathrm{K}_{2} \mathrm{O}$ & $\mathbf{K}$ & $\mathrm{Ca}$ & $\mathrm{Mg}$ & $\mathrm{Fe}$ & $\mathrm{Zn}$ & $\mathrm{Mn}$ & $\mathrm{Cu}$ & \\
\hline & oznaka za SAS & var & rep1 & rep2 & prinos_04 & ST_04 & \begin{tabular}{|l|} 
sirprot_04 \\
\end{tabular} & N_04 & P205_04 & P_04 & K2O_04 & K_04 & Ca_04 & Mg_04 & $\mathrm{Fe} \_04$ & $\mathrm{Zn}$ Z04 & Mn_04 & Cu_04 & Brix_04 \\
\hline 1398 & 1 & 1 & $I$ & 1 & 37,5 & 15,6 & 1,4 & 0,23 & 0,07 & 0,03 & 0,42 & 0,35 & 0,04 & 0,04 & 18,66 & 5,29 & 7,93 & 1,24 & 13,6 \\
\hline 1399 & 2 & 2 & $\mathbf{I}$ & II & 42,7 & 13,0 & 1,3 & 0,20 & 0,09 & 0,04 & 0,44 & 0,37 & 0,03 & 0,03 & 14,29 & 4,29 & 5,20 & 1,23 & 10,5 \\
\hline 1400 & 3 & 3 & $\mathbf{I}$ & III & 44,1 & 15,9 & 1,6 & 0,26 & 0,10 & 0,04 & 0,55 & 0,46 & 0,03 & 0,04 & 15,75 & 5,09 & 7,00 & 1,56 & 12,6 \\
\hline 1401 & 4 & 4 & 1 & IV & 42,9 & 16,0 & 1,5 & 0,24 & 0,09 & 0,04 & 0,52 & 0,43 & 0,04 & 0,03 & 17,23 & 4,47 & 6,54 & 1,75 & 12,9 \\
\hline 1402 & 5 & 2 & II & IV & 46,6 & 13,2 & 1,2 & 0,20 & 0,10 & 0,04 & 0,47 & 0,39 & 0,03 & 0,03 & 15,97 & 3,96 & 4,75 & 1,45 & 10 \\
\hline 1403 & 6 & 4 & II & III & 52,7 & 14,4 & 1,6 & 0,25 & 0,09 & 0,04 & 0,44 & 0,36 & 0,03 & 0,04 & 14,29 & 4,18 & 5,19 & 1,39 & 11,8 \\
\hline 1404 & 7 & 1 & II & II & 43,1 & 14,5 & 1,4 & 0,22 & 0,09 & 0,04 & 0,40 & 0,33 & 0,03 & 0,03 & 13,04 & 4,64 & 5,80 & 1,39 & 12 \\
\hline 1405 & 8 & 3 & II & 1 & 42,4 & 13,4 & 1,5 & 0,25 & 0,08 & 0,03 & 0,40 & 0,33 & 0,03 & 0,03 & 14,50 & 4,97 & 5,51 & 1,32 & 11,6 \\
\hline 1406 & 9 & 2 & III & 1 & 41,1 & 13,5 & 1,2 & 0,19 & 0,04 & 0,02 & 0,37 & 0,31 & 0,02 & 0,03 & 13,33 & 3,77 & 5,38 & 1,12 & 10,5 \\
\hline 1407 & 10 & 4 & III & II & 49,8 & 13,6 & 1,3 & 0,21 & 0,05 & 0,02 & 0,40 & 0,33 & 0,03 & 0,03 & 13,44 & 3,80 & 5,43 & 1,11 & 11,9 \\
\hline 1408 & 11 & 1 & III & III & 47,3 & 13,9 & 1,2 & 0,20 & 0,04 & 0,02 & 0,38 & 0,32 & 0,05 & 0,04 & 16,17 & 3,90 & 5,99 & 1,23 & 12,3 \\
\hline 1409 & 12 & 3 & III & IV & 46,8 & 15,0 & 1,1 & 0,18 & 0,05 & 0,02 & 0,37 & 0,31 & 0,02 & 0,03 & 13,79 & 4,20 & 6,45 & 1,03 & 13,2 \\
\hline 1410 & 13 & 1 & IV & IV & 36,1 & 15,0 & 1,2 & 0,20 & 0,04 & 0,02 & 0,38 & 0,32 & 0,03 & 0,03 & 16,20 & 3,90 & 7,65 & 1,44 & 13,4 \\
\hline 1411 & 14 & 2 & IV & III & 40,0 & 15,9 & 1,4 & 0,22 & 0,04 & 0,02 & 0,43 & 0,36 & 0,03 & 0,04 & 12,69 & 4,12 & 8,25 & 1,44 & 13,8 \\
\hline 1412 & 15 & 3 & IV & II & 43,2 & 15,6 & 1,3 & 0,21 & 0,04 & 0,02 & 0,40 & 0,33 & 0,03 & 0,03 & 14,54 & 4,38 & 7,66 & 1,39 & 13,2 \\
\hline 1413 & 16 & 4 & IV & 1 & 38,1 & 18,3 & 1,5 & 0,24 & 0,04 & 0,02 & 0,42 & 0,35 & 0,04 & 0,03 & 16,44 & 5,12 & 8,95 & 1,61 & 14,7 \\
\hline
\end{tabular}

\begin{tabular}{|c|c|c|c|c|c|c|c|c|c|c|c|c|c|c|c|c|}
\hline \multirow{3}{*}{\multicolumn{2}{|c|}{ Average by variants }} & \multirow{3}{*}{$\begin{array}{l}\text { yield } \\
\text { t/ha }\end{array}$} & \multirow{3}{*}{$\begin{array}{l}\% \\
\text { ST } \\
\end{array}$} & \multirow{3}{*}{\begin{tabular}{|c|} 
crude \\
proteins \\
\end{tabular}} & \multirow{2}{*}{\multicolumn{7}{|c|}{$\%$ in fresh matter }} & \multirow{2}{*}{\multicolumn{4}{|c|}{$\mathrm{mg} / \mathrm{kg}$ in fresh matter }} & \multirow{3}{*}{$\begin{array}{c}\% \\
\text { Brix } \\
\end{array}$} \\
\hline & & & & & & & & & & & & & & & & \\
\hline & & & & & $\mathbf{N}$ & $\mathbf{P}_{2} \mathbf{O}_{5}$ & $\mathbf{P}$ & $\mathrm{K}_{2} \mathrm{O}$ & $\mathbf{K}$ & $\mathrm{Ca}$ & $\mathrm{Mg}$ & $\mathrm{Fe}$ & $\mathrm{Zn}$ & Mn & $\mathrm{Cu}$ & \\
\hline varijant 1 & unfertilized control & 41,0 & 14,7 & 1,3 & 0,2 & 0,06 & 0,03 & 0,4 & 0,3 & 0,0 & 0,0 & 16 & 4 & 7 & 1,3 & 12,8 \\
\hline varijant 2 & stable manure, $50 \mathrm{t} / \mathrm{ha}$ & 42,6 & 13,9 & 1,3 & 0,2 & 0,07 & 0,03 & 0,4 & 0,4 & 0,0 & 0,0 & 14 & 4 & 6 & 1,3 & 11,2 \\
\hline varijant 3 & 500 kg NPK/ha* & 44,1 & 15,0 & 1,4 & 0,2 & 0,07 & 0,03 & 0,4 & 0,4 & 0,0 & 0,0 & 15 & 5 & 7 & 1,3 & 12,7 \\
\hline varijant 4 & 1000 kg NPK/ha* & 45,9 & 15,6 & 1,5 & 0,2 & 0,07 & 0,03 & 0,4 & 0,4 & 0,0 & 0,0 & 15 & 4 & 7 & 1,5 & 12,8 \\
\hline
\end{tabular}




\section{MINERALS IN BEETROOT}

\section{4}

\begin{tabular}{|c|c|c|c|c|c|c|c|c|c|c|c|c|c|c|c|c|c|c|c|}
\hline \multirow[t]{2}{*}{ ID } & \multirow{2}{*}{$\begin{array}{c}\text { sample } \\
\text { label } \\
\end{array}$} & \multirow[b]{2}{*}{ var } & \multirow[b]{2}{*}{ rep } & \multirow[b]{2}{*}{ rep } & \multirow{2}{*}{$\begin{array}{l}\text { yield } \\
\text { t/ha } \\
\end{array}$} & \multirow{2}{*}{$\begin{array}{c}\% \\
\text { ST }\end{array}$} & \multirow{2}{*}{\begin{tabular}{|c|} 
crude \\
proteins \\
\end{tabular}} & \multicolumn{7}{|c|}{$\mathrm{mg} / 100 \mathrm{~g}$ in fresh matter } & \multicolumn{4}{|c|}{$\mathrm{mg} / 100 \mathrm{~g}$ in fresh matter } & \multirow{2}{*}{$\begin{array}{c}\% \\
\text { Brix } \\
\end{array}$} \\
\hline & & & & & & & & $\mathbf{N}$ & $\mathrm{P}_{2} \mathrm{O}_{5}$ & $\mathbf{P}$ & $\mathrm{K}_{2} \mathrm{O}$ & $\mathrm{K}$ & $\mathrm{Ca}$ & $\mathrm{Mg}$ & $\mathrm{Fe}$ & $\mathrm{Zn}$ & $\mathbf{M n}$ & $\mathrm{Cu}$ & \\
\hline & oznaka za SAS & var & rep1 & rep2 & prinos_04 & ST_04 & \begin{tabular}{|l|} 
sirprot_04 \\
\end{tabular} & N_04 & P2O5_04 & P_04 & K2O_04 & K_04 & Ca_04 & Mg_04 & $\mathrm{Fe}_{-} 04$ & $2 n_{-} 04$ & Mn_04 & Cu_04 & Brix_04 \\
\hline 1398 & 1 & 1 & $\mathbf{I}$ & $\mathrm{I}$ & 37,5 & 15,6 & 1418,9 & 227,0 & 71,5 & 31,2 & 418,3 & 348,6 & 38,4 & 38,1 & 1,87 & 0,53 & 0,79 & 0,12 & 13,6 \\
\hline 1399 & 2 & 2 & $\mathbf{I}$ & II & 42,7 & 13,0 & 1258,4 & 201,3 & 89,6 & 39,1 & 439,1 & 365,9 & 27,5 & 25,7 & 1,43 & 0,43 & 0,52 & 0,12 & 10,5 \\
\hline 1400 & 3 & 3 & $I$ & III & 44,1 & 15,9 & 1600,9 & 256,2 & 100,2 & 43,8 & 550,5 & 458,7 & 31,2 & 39,6 & 1,58 & 0,51 & 0,70 & 0,16 & 12,6 \\
\hline 1401 & 4 & 4 & $I$ & IV & 42,9 & 16,0 & 1515,3 & 242,4 & 94,1 & 41,1 & 516,8 & 430,7 & 37,6 & 27,9 & 1,72 & 0,45 & 0,65 & 0,18 & 12,9 \\
\hline 1402 & 5 & 2 & II & IV & 46,6 & 13,2 & 1229,3 & 196,7 & 101,6 & 44,4 & 471,2 & 392,7 & 30,4 & 31,3 & 1,60 & 0,40 & 0,48 & 0,15 & 10 \\
\hline 1403 & 6 & 4 & II & III & 52,7 & 14,4 & 1587,3 & 254,0 & 90,9 & 39,7 & 435,8 & 363,2 & 31,6 & 35,2 & 1,43 & 0,42 & 0,52 & 0,14 & 11,8 \\
\hline 1404 & 7 & 1 & II & II & 43,1 & 14,5 & 1403,7 & 224,6 & 85,5 & 37,3 & 399,9 & 333,3 & 28,8 & 30,4 & 1,30 & 0,46 & 0,58 & 0,14 & 12 \\
\hline 1405 & 8 & 3 & II & 1 & 42,4 & 13,4 & 1536,1 & 245,8 & 77,9 & 34,0 & 397,5 & 331,3 & 26,1 & 29,3 & 1,45 & 0,50 & 0,55 & 0,13 & 11,6 \\
\hline 1406 & 9 & 2 & III & 1 & 41,1 & 13,5 & 1203,0 & 192,5 & 41,2 & 18,0 & 374,2 & 311,8 & 21,5 & 26,9 & 1,33 & 0,38 & 0,54 & 0,11 & 10,5 \\
\hline 1407 & 10 & 4 & III & II & 49,8 & 13,6 & 1341,0 & 214,6 & 46,0 & 20,1 & 395,2 & 329,3 & 25,3 & 25,7 & 1,34 & 0,38 & 0,54 & 0,11 & 11,9 \\
\hline 1408 & 11 & 1 & III & III & 47,3 & 13,9 & 1228,5 & 196,6 & 41,3 & 18,0 & 382,0 & 318,3 & 46,8 & 39,0 & 1,62 & 0,39 & 0,60 & 0,12 & 12,3 \\
\hline 1409 & 12 & 3 & III & IV & 46,8 & 15,0 & 1105,5 & 176,9 & 47,1 & 20,6 & 371,8 & 309,8 & 24,0 & 30,0 & 1,38 & 0,42 & 0,64 & 0,10 & 13,2 \\
\hline 1410 & 13 & 1 & IV & IV & 36,1 & 15,0 & 1237,5 & 198,0 & 38,3 & 16,7 & 384,0 & 320,0 & 31,5 & 30,0 & 1,62 & 0,39 & 0,77 & 0,14 & 13,4 \\
\hline 1411 & 14 & 2 & IV & III & 40,0 & 15,9 & 1358,0 & 217,3 & 44,9 & 19,6 & 428,2 & 356,9 & 30,6 & 35,7 & 1,27 & 0,41 & 0,82 & 0,14 & 13,8 \\
\hline 1412 & 15 & 3 & IV & II & 43,2 & 15,6 & 1309,0 & 209,4 & 43,5 & 19,0 & 401,7 & 334,7 & 28,1 & 28,1 & 1,45 & 0,44 & 0,77 & 0,14 & 13,2 \\
\hline 1413 & 16 & 4 & IV & 1 & 38,1 & 18,3 & 1530,1 & 244,8 & 44,6 & 19,5 & 418,4 & 348,7 & 38,4 & 34,5 & 1,64 & 0,51 & 0,90 & 0,16 & 14,7 \\
\hline
\end{tabular}

\section{Average by variants}

\begin{tabular}{|c|l|}
\hline varijant 1 & unfertilized control \\
\hline
\end{tabular}

varijant 2 stable manure, $50 \mathrm{t} / \mathrm{ha}$

\begin{tabular}{l|l} 
varijant 3 & $500 \mathrm{~kg} \mathrm{NPK} / \mathrm{ha}^{*}$
\end{tabular}

\begin{tabular}{|l|l|}
\hline varijant 4 & $1000 \mathrm{~kg} \mathrm{NPK} / \mathrm{ha}^{*}$ \\
\hline
\end{tabular}

\begin{tabular}{|c|c|c|c|c|c|c|c|c|c|c|c|c|c|c|}
\hline $\begin{array}{l}\text { yield } \\
\text { yis }\end{array}$ & $\%$ & crude & \multicolumn{7}{|c|}{$\mathrm{mg} / 100 \mathrm{~g}$ in fresh matter } & \multicolumn{4}{|c|}{$\mathrm{mg} / 100 \mathrm{~g}$ in fresh matter } & $\%$ \\
\hline t/ha & ST & proteins & $\mathbf{N}$ & $\mathrm{P}_{2} \mathrm{O}_{5}$ & $\mathbf{P}$ & $\mathrm{K}_{2} \mathrm{O}$ & $\mathrm{K}$ & $\mathrm{Ca}$ & $\mathrm{Mg}$ & $\mathrm{Fe}$ & $\mathrm{Zn}$ & $M n$ & $\mathrm{Cu}$ & Brix \\
\hline 41,0 & 14,7 & 1322 & 212 & 59 & 26 & 396 & 330 & 36 & 34 & 1,60 & 0,44 & 0,68 & 0,13 & 12,8 \\
\hline 42,6 & 13,9 & 1262 & 202 & 69 & 30 & 428 & 357 & 28 & 30 & 1,41 & 0,40 & 0,59 & 0,13 & 11,2 \\
\hline 44,1 & 15,0 & 1388 & 222 & 67 & 29 & 430 & 359 & 27 & 32 & 1,46 & 0,47 & 0,67 & 0,13 & 12,7 \\
\hline 45,9 & 15,6 & 1493 & 239 & 69 & 30 & 442 & 368 & 33 & 31 & 1,53 & 0,44 & 0,65 & 0,15 & 12,8 \\
\hline
\end{tabular}




\section{MINERALS IN BEETROOT}

\section{5}

\begin{tabular}{|c|c|c|c|c|c|c|c|c|c|c|c|c|c|c|c|c|c|c|c|}
\hline \multirow[t]{2}{*}{ ID } & \multirow{2}{*}{$\begin{array}{c}\text { sample } \\
\text { label } \\
\end{array}$} & \multirow[b]{2}{*}{ var } & \multirow[b]{2}{*}{ rep } & \multirow[b]{2}{*}{ rep } & \multirow{2}{*}{$\begin{array}{l}\text { yield } \\
\text { t/ha }\end{array}$} & \multirow{2}{*}{$\begin{array}{c}\% \\
\text { ST } \\
\end{array}$} & \multirow{2}{*}{\begin{tabular}{|c|} 
crude \\
proteins \\
\end{tabular}} & \multicolumn{7}{|c|}{$\%$ in dry matter } & \multicolumn{4}{|c|}{$\mathrm{mg} / \mathrm{kg}$ in dry matter } & \multirow{2}{*}{$\begin{array}{c}\% \\
\text { Brix } \\
\end{array}$} \\
\hline & & & & & & & & $\mathbf{N}$ & $\mathrm{P}_{2} \mathrm{O}_{5}$ & $\mathbf{P}$ & $\mathrm{K}_{2} \mathrm{O}$ & $K$ & $\mathrm{Ca}$ & $\mathrm{Mg}$ & $\mathrm{Fe}$ & $\mathrm{Zn}$ & $\mathrm{Mn}$ & $\mathrm{Cu}$ & \\
\hline & oznaka za SAS & var & rep1 & rep2 & prinos_05 & ST_05 & sirprot_05 & N_05 & P205_05 & P_05 & K2O_05 & K_05 & Ca_05 & Mg_05 & $\mathrm{Fe} \_05$ & $\mathrm{Zn} \_05$ & Mn_05 & Cu_05 & Brix_05 \\
\hline 3250 & 1 & 1 & 1 & 1 & 8,0 & 7,22 & 19,7 & 3,15 & 0,893 & 0,39 & 5,07 & 4,23 & 0,296 & 0,25 & 96,0 & 45,0 & 66,0 & 5,4 & 11,6 \\
\hline 3251 & 2 & 2 & $\mathbf{I}$ & II & 15,0 & 6,31 & 21,0 & 3,36 & 0,829 & 0,36 & 6,43 & 5,36 & 0,254 & 0,27 & 99,0 & 43,0 & 55,0 & 6,0 & 10,0 \\
\hline 3252 & 3 & 3 & $\mathbf{I}$ & III & 22,2 & 6,59 & 18,7 & 2,99 & 0,89 & 0,39 & 6,24 & 5,20 & 0,24 & 0,23 & 96,0 & 42,0 & 60,0 & 4,8 & 10,4 \\
\hline 3253 & 4 & 4 & 1 & IV & 9,5 & 6,33 & 20,6 & 3,3 & 0,89 & 0,39 & 6,28 & 5,23 & 0,283 & 0,27 & 105,0 & 40,0 & 62,0 & 5,4 & 8,9 \\
\hline 3254 & 5 & 2 & II & IV & 14,9 & 6,28 & 20,0 & 3,2 & 1,074 & 0,47 & 6,24 & 5,20 & 0,27 & 0,24 & 109,0 & 41,0 & 56,0 & 5,6 & 9,9 \\
\hline 3255 & 6 & 4 & II & III & 26,4 & 6,10 & 21,4 & 3,42 & 1,07 & 0,47 & 6,44 & 5,37 & 0,27 & 0,25 & 99,0 & 38,0 & 59,0 & 5,0 & 8,4 \\
\hline 3256 & 7 & 1 & II & II & 22,8 & 6,31 & 18,5 & 2,96 & 0,81 & 0,35 & 5,8 & 4,83 & 0,25 & 0,22 & 92,0 & 40,0 & 55,0 & 5,6 & 10,3 \\
\hline 3257 & 8 & 3 & II & 1 & 20,3 & 6,01 & 18,7 & 2,99 & 0,848 & 0,37 & 5,55 & 4,63 & 0,23 & 0,23 & 98,0 & 44,0 & 56,0 & 5,2 & 10,2 \\
\hline 3258 & 9 & 2 & III & 1 & 32,7 & 5,88 & 18,9 & 3,02 & 1,09 & 0,48 & 5,01 & 4,18 & 0,22 & 0,26 & 95,0 & 39,0 & 56,0 & 4,6 & 9,6 \\
\hline 3259 & 10 & 4 & III & II & 38,1 & 7,28 & 21,0 & 3,36 & 0,729 & 0,32 & 5,54 & 4,62 & 0,231 & 0,26 & 90,0 & 39,0 & 59,0 & 5,6 & 10,7 \\
\hline 3260 & 11 & 1 & III & III & 28,6 & 7,91 & 21,4 & 3,42 & 1,00 & 0,44 & 4,95 & 4,13 & 0,289 & 0,24 & 101,0 & 40,0 & 60,0 & 5,6 & 10,4 \\
\hline 3261 & 12 & 3 & III & IV & 15,0 & 8,3 & 18,4 & 2,94 & 0,63 & 0,28 & 5,07 & 4,23 & 0,24 & 0,23 & 90,0 & 39,0 & 55,0 & 5,1 & 11,2 \\
\hline 3262 & 13 & 1 & IV & IV & 10,9 & 7,78 & 17,9 & 2,87 & 0,66 & 0,29 & 5,54 & 4,62 & 0,256 & 0,23 & 98,0 & 39,0 & 66,0 & 5,4 & 11,2 \\
\hline 3263 & 14 & 2 & IV & III & 39,6 & 6,66 & 19,4 & 3,1 & 0,67 & 0,29 & 5,29 & 4,41 & 0,251 & 0,23 & 84,0 & 38,0 & 62,0 & 5,4 & 9,3 \\
\hline 3264 & 15 & 3 & IV & II & 38,0 & 7,23 & 19,6 & 3,14 & 0,78 & 0,34 & 5,07 & 4,23 & 0,292 & 0,26 & 89,0 & 39,0 & 59,0 & 5,1 & 10,6 \\
\hline \multirow[t]{2}{*}{3265} & 16 & 4 & IV & 1 & 29,3 & 9,45 & 20,4 & 3,27 & 0,73 & 0,32 & 4,66 & 3,88 & 0,259 & 0,2 & 92,0 & 38,0 & 60,0 & 5,0 & 10,8 \\
\hline & & & & & yield & $\%$ & crude & \multicolumn{7}{|c|}{$\%$ in dry matter } & \multicolumn{4}{|c|}{$\mathrm{mg} / \mathrm{kg}$ in dry matter } & $\%$ \\
\hline \multicolumn{3}{|c|}{ Average by variants } & & & t/ha & ST & proteins & $\mathbf{N}$ & $\mathrm{P}_{2} \mathrm{O}_{5}$ & $\mathbf{P}$ & $\mathrm{K}_{2} \mathrm{O}$ & $K$ & $\mathrm{Ca}$ & $\mathrm{Mg}$ & $\mathrm{Fe}$ & $\mathrm{Zn}$ & $\mathrm{Mn}$ & $\mathrm{Cu}$ & Brix \\
\hline varijant 1 & \multirow{2}{*}{\multicolumn{3}{|c|}{\begin{tabular}{|l|}
$\mid$ unfertilized control \\
stable manure, $50 \mathrm{t} / \mathrm{ha}$
\end{tabular}}} & & 17,6 & 7,3 & 19,4 & 3,1 & 0,8 & 0,4 & 5,3 & 4,5 & 0,3 & 0,2 & 96,8 & 41,0 & 61,8 & 5,5 & 10,9 \\
\hline varijant 2 & & & & & 25,6 & 6,3 & 19,8 & 3,2 & 0,9 & 0,4 & 5,7 & 4,8 & 0,2 & 0,3 & 96,8 & 40,3 & 57,3 & 5,4 & 9,7 \\
\hline varijant 3 & \multicolumn{2}{|c|}{500 kg NPK/ha* } & & & 23,9 & 7,0 & 18,8 & 3,0 & 0,8 & 0,3 & 5,5 & 4,6 & 0,3 & 0,2 & 93,3 & 41,0 & 57,5 & 5,0 & 10,6 \\
\hline varijant 4 & \multicolumn{2}{|c|}{1000 kg NPK/ha* } & & & 25,8 & 7,3 & 20,9 & 3,3 & 0,9 & 0,4 & 5,7 & 4,8 & 0,3 & 0,2 & 96,5 & 38,8 & 60,0 & 5,3 & 9,7 \\
\hline
\end{tabular}




\section{MINERALS IN BEETROOT}

\section{5}

\begin{tabular}{|c|c|c|c|c|c|c|c|c|c|c|c|c|c|c|c|c|c|c|c|}
\hline \multirow[t]{2}{*}{ ID } & \multirow{2}{*}{$\begin{array}{c}\text { sample } \\
\text { label }\end{array}$} & \multirow[b]{2}{*}{ var } & \multirow[b]{2}{*}{ rep } & \multirow[b]{2}{*}{ rep } & \multirow{2}{*}{$\begin{array}{l}\text { yield } \\
\mathrm{t} / \mathrm{ha} \\
\end{array}$} & \multirow{2}{*}{$\begin{array}{c}\% \\
\text { ST } \\
\end{array}$} & \multirow{2}{*}{\begin{tabular}{|c|} 
crude \\
proteins \\
\end{tabular}} & \multicolumn{7}{|c|}{$\%$ in fresh matter } & \multicolumn{4}{|c|}{$\mathrm{mg} / \mathrm{kg}$ in fresh matter } & \multirow{2}{*}{$\begin{array}{c}\% \\
\text { Brix } \\
\end{array}$} \\
\hline & & & & & & & & $\mathbf{N}$ & $\mathbf{P}_{2} \mathbf{O}_{5}$ & $\mathbf{P}$ & $\mathrm{K}_{2} \mathrm{O}$ & $\mathbf{K}$ & $\mathrm{Ca}$ & $\mathrm{Mg}$ & $\mathrm{Fe}$ & $\mathrm{Zn}$ & $\mathrm{Mn}$ & $\mathrm{Cu}$ & \\
\hline & oznaka za SAS & var & rep1 & rep2 & prinos_05 & ST_05 & sirprot_05 & N_05 & P205_05 & P_05 & K2O_05 & K_05 & Ca_05 & Mg_05 & $\mathrm{Fe} \_05$ & $2 n \_05$ & Mn_05 & $\mathrm{Cu}_{-} 05$ & Brix_05 \\
\hline 1398 & 1 & 1 & $I$ & 1 & 8,0 & 7,22 & 1,4 & 0,23 & 0,06 & 0,03 & 0,37 & 0,31 & 0,02 & 0,02 & 6,93 & 3,25 & 4,77 & 0,39 & 11,6 \\
\hline 1399 & 2 & 2 & $\mathbf{I}$ & II & 15,0 & 6,31 & 1,3 & 0,21 & 0,05 & 0,02 & 0,41 & 0,34 & 0,02 & 0,02 & 6,25 & 2,71 & 3,47 & 0,38 & 10,0 \\
\hline 1400 & 3 & 3 & $\mathbf{I}$ & III & 22,2 & 6,59 & 1,2 & 0,20 & 0,06 & 0,03 & 0,41 & 0,34 & 0,02 & 0,02 & 6,33 & 2,77 & 3,95 & 0,32 & 10,4 \\
\hline 1401 & 4 & 4 & 1 & IV & 9,5 & 6,33 & 1,3 & 0,21 & 0,06 & 0,02 & 0,40 & 0,33 & 0,02 & 0,02 & 6,65 & 2,53 & 3,92 & 0,34 & 8,9 \\
\hline 1402 & 5 & 2 & II & IV & 14,9 & 6,28 & 1,3 & 0,20 & 0,07 & 0,03 & 0,39 & 0,33 & 0,02 & 0,02 & 6,85 & 2,57 & 3,52 & 0,35 & 9,9 \\
\hline 1403 & 6 & 4 & II & III & 26,4 & 6,10 & 1,3 & 0,21 & 0,07 & 0,03 & 0,39 & 0,33 & 0,02 & 0,02 & 6,04 & 2,32 & 3,60 & 0,31 & 8,4 \\
\hline 1404 & 7 & 1 & II & II & 22,8 & 6,31 & 1,2 & 0,19 & 0,05 & 0,02 & 0,37 & 0,30 & 0,02 & 0,01 & 5,81 & 2,52 & 3,47 & 0,35 & 10,3 \\
\hline 1405 & 8 & 3 & II & 1 & 20,3 & 6,01 & 1,1 & 0,18 & 0,05 & 0,02 & 0,33 & 0,28 & 0,01 & 0,01 & 5,89 & 2,64 & 3,37 & 0,31 & 10,2 \\
\hline 1406 & 9 & 2 & III & 1 & 32,7 & 5,88 & 1,1 & 0,18 & 0,06 & 0,03 & 0,29 & 0,25 & 0,01 & 0,02 & 5,59 & 2,29 & 3,29 & 0,27 & 9,6 \\
\hline 1407 & 10 & 4 & III & II & 38,1 & 7,28 & 1,5 & 0,24 & 0,05 & 0,02 & 0,40 & 0,34 & 0,02 & 0,02 & 6,55 & 2,84 & 4,30 & 0,41 & 10,7 \\
\hline 1408 & 11 & 1 & III & III & 28,6 & 7,91 & 1,7 & 0,27 & 0,08 & 0,03 & 0,39 & 0,33 & 0,02 & 0,02 & 7,99 & 3,16 & 4,75 & 0,44 & 10,4 \\
\hline 1409 & 12 & 3 & III & IV & 15,0 & 8,3 & 1,5 & 0,24 & 0,05 & 0,02 & 0,42 & 0,35 & 0,02 & 0,02 & 7,47 & 3,24 & 4,57 & 0,42 & 11,2 \\
\hline 1410 & 13 & 1 & IV & IV & 10,9 & 7,78 & 1,4 & 0,22 & 0,05 & 0,02 & 0,43 & 0,36 & 0,02 & 0,02 & 7,62 & 3,03 & 5,13 & 0,42 & 11,2 \\
\hline 1411 & 14 & 2 & IV & III & 39,6 & 6,66 & 1,3 & 0,21 & 0,04 & 0,02 & 0,35 & 0,29 & 0,02 & 0,02 & 5,59 & 2,53 & 4,13 & 0,36 & 9,3 \\
\hline 1412 & 15 & 3 & IV & II & 38,0 & 7,23 & 1,4 & 0,23 & 0,06 & 0,02 & 0,37 & 0,31 & 0,02 & 0,02 & 6,43 & 2,82 & 4,27 & 0,37 & 10,6 \\
\hline 1413 & 16 & 4 & IV & 1 & 29,3 & 9,45 & 1,9 & 0,31 & 0,07 & 0,03 & 0,44 & 0,37 & 0,02 & 0,02 & 8,69 & 3,59 & 5,67 & 0,47 & 10,8 \\
\hline
\end{tabular}

\begin{tabular}{|c|c|c|c|c|c|c|c|c|c|c|c|c|c|c|c|c|}
\hline \multirow{3}{*}{\multicolumn{2}{|c|}{ Average by variants }} & \multirow{3}{*}{\multicolumn{2}{|c|}{\begin{tabular}{c|c} 
yield & $\%$ \\
t/ha & ST \\
\end{tabular}}} & \multirow{3}{*}{\begin{tabular}{|c|} 
crude \\
proteins \\
\end{tabular}} & \multirow{2}{*}{\multicolumn{7}{|c|}{$\%$ in fresh matter }} & \multirow{2}{*}{\multicolumn{4}{|c|}{$\mathrm{mg} / \mathrm{kg}$ in fresh matter }} & \multirow{3}{*}{$\begin{array}{c}\% \\
\text { Brix }\end{array}$} \\
\hline & & & & & & & & & & & & & & & & \\
\hline & & & & & $\mathbf{N}$ & $\mathrm{P}_{2} \mathrm{O}_{5}$ & $\mathbf{P}$ & $\mathrm{K}_{2} \mathrm{O}$ & $\mathrm{K}$ & $\mathrm{Ca}$ & $\mathrm{Mg}$ & $\mathrm{Fe}$ & $\mathrm{Zn}$ & $\mathrm{Mn}$ & $\mathrm{Cu}$ & \\
\hline varijant 1 & unfertilized control & 17,6 & 7,3 & 1,4 & 0,2 & 0,06 & 0,03 & 0,4 & 0,3 & 0,020 & 0,017 & 7 & 3 & 5 & 0,4 & 10,9 \\
\hline varijant 2 & stable manure, $50 \mathrm{t} / \mathrm{ha}$ & 25,6 & 6,3 & 1,2 & 0,2 & 0,06 & 0,02 & 0,4 & 0,3 & 0,016 & 0,016 & 6 & 3 & 4 & 0,3 & 9,7 \\
\hline varijant 3 & 500 kg NPK/ha* & 23,9 & 7,0 & 1,3 & 0,2 & 0,05 & 0,02 & 0,4 & 0,3 & 0,018 & 0,017 & 7 & 3 & 4 & 0,4 & 10,6 \\
\hline varijant 4 & 1000 kg NPK/ha* & 25,8 & 7,3 & 1,5 & 0,2 & 0,06 & 0,03 & 0,4 & 0,3 & 0,019 & 0,018 & 7 & 3 & 4 & 0,4 & 9,7 \\
\hline
\end{tabular}




\section{MINERALS IN BEETROOT}

\section{5}

\begin{tabular}{|c|c|c|c|c|c|c|c|c|c|c|c|c|c|c|c|c|c|c|c|}
\hline \multirow[t]{2}{*}{ ID } & \multirow{2}{*}{$\begin{array}{c}\text { sample } \\
\text { label }\end{array}$} & \multirow[b]{2}{*}{ var } & \multirow[b]{2}{*}{ rep } & \multirow[b]{2}{*}{ rep } & \multirow{2}{*}{$\begin{array}{l}\text { yield } \\
\mathrm{t} / \mathrm{ha} \\
\end{array}$} & \multirow{2}{*}{$\begin{array}{c}\% \\
\text { ST } \\
\end{array}$} & \multirow{2}{*}{\begin{tabular}{|c|} 
crude \\
proteins \\
\end{tabular}} & \multicolumn{7}{|c|}{$\mathrm{mg} / 100 \mathrm{~g}$ in fresh matter } & \multicolumn{4}{|c|}{$\mathrm{mg} / 100 \mathrm{~g}$ in fresh matter } & \multirow{2}{*}{$\begin{array}{c}\% \\
\text { Brix } \\
\end{array}$} \\
\hline & & & & & & & & $\mathbf{N}$ & $\mathbf{P}_{2} \mathbf{O}_{5}$ & $\mathbf{P}$ & $\mathrm{K}_{2} \mathrm{O}$ & $\mathbf{K}$ & $\mathrm{Ca}$ & $\mathrm{Mg}$ & $\mathrm{Fe}$ & $\mathrm{Zn}$ & $\mathrm{Mn}$ & $\mathrm{Cu}$ & \\
\hline & oznaka za SAS & var & rep1 & rep2 & prinos_05 & ST_05 & sirprot_05 & N_05 & P2O5_05 & P_05 & K2O_05 & K_05 & Ca_05 & Mg_05 & $\mathrm{Fe} \_05$ & $2 n$ & Mn_05 & $\mathrm{Cu}_{-} 05$ & Brix_05 \\
\hline 1398 & 1 & 1 & $\mathbf{I}$ & 1 & 8,0 & 7,22 & 1421,4 & 227,4 & 64,5 & 28,2 & 366,1 & 305,0 & 21,4 & 18,1 & 0,69 & 0,32 & 0,48 & 0,04 & 11,6 \\
\hline 1399 & 2 & 2 & $\mathbf{I}$ & II & 15,0 & 6,31 & 1325,1 & 212,0 & 52,3 & 22,8 & 405,7 & 338,1 & 16,0 & 17,0 & 0,62 & 0,27 & 0,35 & 0,04 & 10,0 \\
\hline 1400 & 3 & 3 & 1 & III & 22,2 & 6,59 & 1231,5 & 197,0 & 58,7 & 25,6 & 411,2 & 342,7 & 15,8 & 15,2 & 0,63 & 0,28 & 0,40 & 0,03 & 10,4 \\
\hline 1401 & 4 & 4 & 1 & IV & 9,5 & 6,33 & 1305,6 & 208,9 & 56,3 & 24,6 & 397,5 & 331,3 & 17,9 & 17,1 & 0,66 & 0,25 & 0,39 & 0,03 & 8,9 \\
\hline 1402 & 5 & 2 & II & IV & 14,9 & 6,28 & 1256,0 & 201,0 & 67,4 & 29,5 & 391,9 & 326,6 & 17,0 & 15,1 & 0,68 & 0,26 & 0,35 & 0,04 & 9,9 \\
\hline 1403 & 6 & 4 & II & III & 26,4 & 6,10 & 1303,9 & 208,6 & 65,3 & 28,5 & 392,8 & 327,4 & 16,5 & 15,3 & 0,60 & 0,23 & 0,36 & 0,03 & 8,4 \\
\hline 1404 & 7 & 1 & II & II & 22,8 & 6,31 & 1167,4 & 186,8 & 51,1 & 22,3 & 366,0 & 305,0 & 15,8 & 13,9 & 0,58 & 0,25 & 0,35 & 0,04 & 10,3 \\
\hline 1405 & 8 & 3 & II & 1 & 20,3 & 6,01 & 1123,1 & 179,7 & 51,0 & 22,3 & 333,6 & 278,0 & 13,8 & 13,8 & 0,59 & 0,26 & 0,34 & 0,03 & 10,2 \\
\hline 1406 & 9 & 2 & III & 1 & 32,7 & 5,88 & 1109,9 & 177,6 & 64,1 & 28,0 & 294,6 & 245,5 & 12,9 & 15,3 & 0,56 & 0,23 & 0,33 & 0,03 & 9,6 \\
\hline 1407 & 10 & 4 & III & II & 38,1 & 7,28 & 1528,8 & 244,6 & 53,1 & 23,2 & 403,3 & 336,1 & 16,8 & 18,9 & 0,66 & 0,28 & 0,43 & 0,04 & 10,7 \\
\hline 1408 & 11 & 1 & III & III & 28,6 & 7,91 & 1690,8 & 270,5 & 79,1 & 34,5 & 391,5 & 326,3 & 22,9 & 19,0 & 0,80 & 0,32 & 0,47 & 0,04 & 10,4 \\
\hline 1409 & 12 & 3 & III & IV & 15,0 & 8,3 & 1525,1 & 244,0 & 52,3 & 22,8 & 420,8 & 350,7 & 19,9 & 19,1 & 0,75 & 0,32 & 0,46 & 0,04 & 11,2 \\
\hline 1410 & 13 & 1 & IV & IV & 10,9 & 7,78 & 1395,5 & 223,3 & 51,3 & 22,4 & 431,0 & 359,2 & 19,9 & 17,9 & 0,76 & 0,30 & 0,51 & 0,04 & 11,2 \\
\hline 1411 & 14 & 2 & IV & III & 39,6 & 6,66 & 1290,4 & 206,5 & 44,6 & 19,5 & 352,3 & 293,6 & 16,7 & 15,3 & 0,56 & 0,25 & 0,41 & 0,04 & 9,3 \\
\hline 1412 & 15 & 3 & IV & II & 38,0 & 7,23 & 1418,9 & 227,0 & 56,4 & 24,6 & 366,6 & 305,5 & 21,1 & 18,8 & 0,64 & 0,28 & 0,43 & 0,04 & 10,6 \\
\hline 1413 & 16 & 4 & IV & 1 & 29,3 & 9,45 & 1931,3 & 309,0 & 69,0 & 30,1 & 440,4 & 367,0 & 24,5 & 18,9 & 0,87 & 0,36 & 0,57 & 0,05 & 10,8 \\
\hline
\end{tabular}

\begin{tabular}{|c|c|c|c|c|c|c|c|c|c|c|c|c|c|c|c|c|}
\hline & & \multirow[b]{2}{*}{ yield } & \multirow{3}{*}{\multicolumn{2}{|c|}{\begin{tabular}{|c|} 
crude \\
proteins \\
\end{tabular}}} & \multirow{2}{*}{\multicolumn{7}{|c|}{$\mathrm{mg} / 100 \mathrm{~g}$ in fresh matter }} & \multirow{2}{*}{\multicolumn{4}{|c|}{$\mathrm{mg} / 100 \mathrm{~g}$ in fresh matter }} & \multirow{3}{*}{\begin{tabular}{|c|}
$\%$ \\
Brix \\
\end{tabular}} \\
\hline & & & & & & & & & & & & & & & & \\
\hline \multicolumn{2}{|c|}{ Average by variants } & t/ha & & & $\mathbf{N}$ & $\mathbf{P}_{2} \mathbf{O}_{5}$ & $\mathbf{P}$ & $\mathrm{K}_{2} \mathrm{O}$ & $\mathbf{K}$ & $\mathrm{Ca}$ & $\mathrm{Mg}$ & $\mathrm{Fe}$ & $\mathrm{Zn}$ & $\mathrm{Mn}$ & $\mathrm{Cu}$ & \\
\hline varijant 1 & unfertilized control & 17,6 & 7,3 & 1419 & 227 & 62 & 27 & 389 & 324 & 20 & 17 & 0,71 & 0,30 & 0,45 & 0,04 & 10,9 \\
\hline varijant 2 & stable manure, $50 \mathrm{t} / \mathrm{ha}$ & 25,6 & 6,3 & 1245 & 199 & 57 & 25 & 361 & 301 & 16 & 16 & 0,61 & 0,25 & 0,36 & 0,03 & 9,7 \\
\hline varijant 3 & 500 kg NPK/ha* & 23,9 & 7,0 & 1325 & 212 & 55 & 24 & 383 & 319 & 18 & 17 & 0,65 & 0,29 & 0,40 & 0,04 & 10,6 \\
\hline varijant 4 & 1000 kg NPK/ha* & 25,8 & 7,3 & 1517 & 243 & 61 & 27 & 409 & 340 & 19 & 18 & 0,70 & 0,28 & 0,44 & 0,04 & 9,7 \\
\hline
\end{tabular}

